\title{
SUPER-CRITICAL REFLECTION OF OCEAN WAVES; A NEW FACTOR IN ICE-EDGE DYNAMICS?
}

\author{
by \\ Vernon A. Squire \\ (Department of Mathematics and Statistics, University of Otago, P.O. Box 56, \\ Dunedin, New Zealand)
}

\section{ABSTRACT}

Movement of the sea-ice edge on short time-scales $(<1 \mathrm{~d})$ is due to a balance of forces between several mechanisms (wind stress, sea-surface tilt, internal ice stress, and Coriolis force) which are often comparable in magnitude. Other factors such as the force induced by partial reflection of short seas, internal gravity waves in the pycnocline, etc., may also contribute. Through the momentum equation, these mechanisms affect the dynamics of the ice edge. In this paper we suggest another mechanism which may have importance, namely, a radiation-stress contribution which derives from obliquely incident waves which are totally reflected from the ice edge by a process analogous to total internal reflection in optics. Such reflection generates both normal and shear forces at the ice edge, the former tending to compact the pack ice and the latter to shear the absolute edge. The effect is studied using some recent data collected during the Winter Weddell Sea Project 1986 in Antarctica, where it is found that the contribution to the force balance is significant. For thicker sea ice and icebergs acted upon by oblique seas, the radiation stress-induced force may outweigh more conventional terms in the momentum equation.

\section{INTRODUCTION}

The precise nature of the momentum balance at the ice edge is rather poorly understood; for as well as the usual balance of forces due to wind stress, water stress, sea-surface tilt, internal ice stress, and Coriolis force, other factors which owe their existence to the proximity of the open ocean are known to be important. These include a radiation-stress mechanism known to create and to keep ice bands intact (Martin and others, 1983; Wadhams, 1983); an internal gravity-wave mechanism (Muench and others, 1983) which leads to zones of convergence and divergence, and hence also to banding of the ice cover; spatial changes in both the air-ice and water-ice drag coefficients; and numerous mechanisms which rely on modification of oceanographic structure induced by the (often abrupt) change from an open to an ice-covered ocean.

In this paper we investigate another aspect of ocean waves which may have importance to the driving of the ice edge. This is the mechanism of total external reflection of surface waves, first postulated by Squire (unpublished) and shown theoretically possible for a shore-fast ice edge and large ice floes by Squire (1984). When such reflection does occur, the radiation stress tensor at the ice edge has a nonzero normal element and a non-zero shear element. Both may be significant in typical ice-edge seas. This paper develops the appropriate theory and investigates the magnitude of the forces induced by the phenomenon in a case study.

\section{THE CRITICAL ANGLE FOR TOTAL REFLECTION}

Consider a train of deep-ocean surface waves incident on an ice edge obliquely. On the open ocean side of the ice edge the dispersion equation for the waves is

$$
k=\frac{\omega^{2}}{g}
$$

where $k$ and $\omega$ are respectively the wave number and radian frequency of the incident wave, and $g$ is the acceleration due to gravity. As usual, $k=2 \pi /$ wavelength and $\omega=2 \pi /$ period.

We assume that the ice cover may be modelled as a thin elastic plate. This is an approximation which may hold under certain circumstances; namely, where ice floes are large compared to wavelength or where discrete floes have become sufficiently abraded and pummelled together by wave action to create a zone of $100 \%$ concentration at the ice edge. A dispersion relation may then be written down for the ice cover as follows:

$$
D k^{\prime 5}+\left(\rho g-\rho^{\prime} h \omega^{2}\right) k^{\prime}-\rho \omega^{2}=0
$$

where $k^{\prime}$ is the wave number in ice, $D$ is the flexural rigidity of the sea ice, $\rho$ is sea-water density, $\rho^{\prime}$ is the ice density, and $h$ is the ice thickness. The flexural rigidity is defined as $E h^{3} / 12\left(1-v^{2}\right)$ where $E$ is Young's modulus and $U$ is Poisson's ratio. Relation (2) is found from the elastic thin-plate equation, the kinematic boundary condition beneath the ice, and Bernoulli's pressure equation. For a full derivation see Squire (1984). Following that paper further, we assume oblique incidence by considering a surface-wave displacement $n$ in the open sea of the form

$\eta=\left[I \mathrm{e}^{i k(x \cos \theta+y \sin \theta)}+R \mathrm{e}^{-i k(x \cos \theta-y \sin \theta)}\right] \mathrm{e}^{-i \omega t}$.

In this expression $(x, y)$ is a coordinate system with $x$ normal and into the ice edge and $y$ along the ice edge, $\theta$ is the angle of incidence, and $I$ and $R$ are the incident and reflected wave amplitudes. A wave displacement of similar form to Equation (3) exists within the ice cover. By appropriate matching across the ice edge, we find an expression analogous to Snell's Law of optics, viz.

$$
k \sin \theta=k^{\prime} \sin \theta^{\prime}
$$

where $\theta^{\prime}$ is the angle of refraction. We now ask the question: do any angles of incidence exist for which Equation (4) cannot be satisfied? Clearly, this depends on the relative magnitudes of $k$ and $k^{\prime}$, since if $k \sin \theta>k^{\prime}$ no real angle $\theta^{\prime}$ can be found. This suggests that a critical angle of incidence $\theta_{\mathrm{c}}$ can be identified when $\sin \theta_{\mathrm{c}}=k^{\prime} / k$. For angles of incidence less than $\theta_{c}$ the waves penetrate the ice; for angles greater than $\theta_{c}$ no propagating wave is permitted to enter. It is known for representative values of the elastic parameters $E$ and $v$ that $k^{\prime}<k$ for short waves and $k^{\prime}>k$ for long waves, and that the greater the ice thickness the lower the period of transition (Squire, unpublished). Using typical elastic constants for sea ice $\left(E=6.0 \times 10^{9} \mathrm{~N} \mathrm{~m}^{-2}\right.$ and $\left.v=0.3\right)$, the critical angle is 


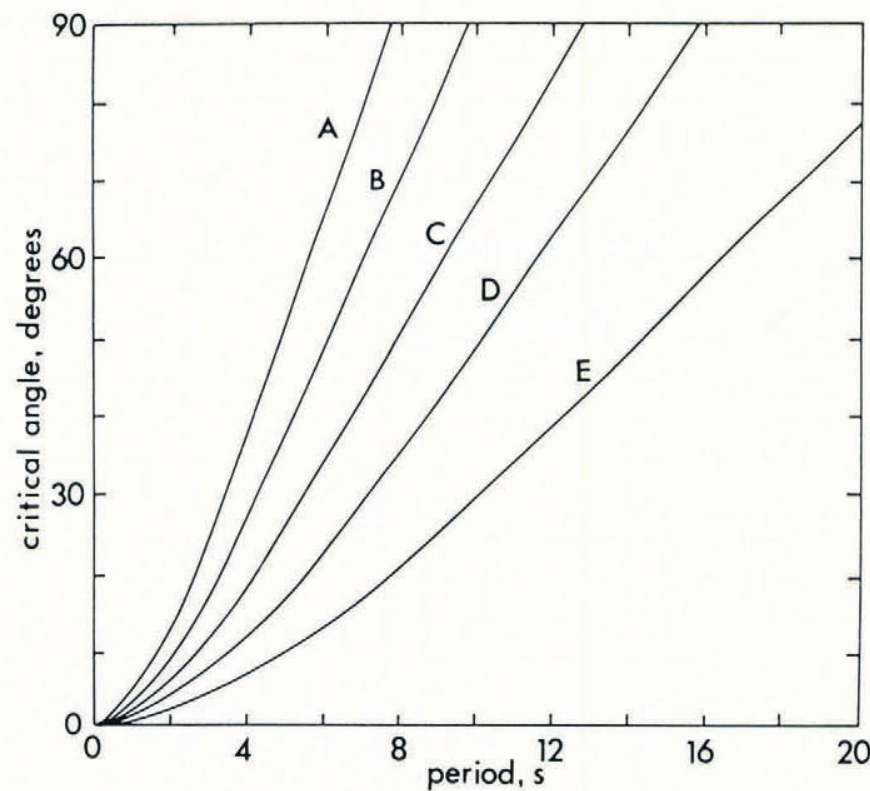

Fig. 1. Critical angle curves for various ice thicknesses plotted as a function of wave period: A, $0.25 \mathrm{~m} ; \mathrm{B}, 0.5 \mathrm{~m}$; C, $1.0 \mathrm{~m} ; \mathrm{D}, \quad 2.0 \mathrm{~m} ; \mathrm{E}, \quad 5.0 \mathrm{~m}$. Reproduced with modification from Squire (1984).

plotted at various thicknesses as a function of period in Figure 1. Total external reflection occurs at incidence angles above those defined by the intersection of the appropriate thickness curve and the period ordinate. With such reflection, the amplitude of the reflected wave will be the same as the incident wave. Thus, the sea off the ice edge will take on the form of a wave travelling along the edge whose amplitude is modulated by a standing pattern of corrugations with nodal and anti-nodal lines parallel to the edge. Most important to this discussion: reflection is complete, i.e. $|R| /|I|=1$.

\section{RADIATION STRESS}

The theory of radiation stress in water waves is well understood and the reader is referred to Longuet-Higgins and Stewart (1964) for a complete treatment, and to Longuet-Higgins (1977) for an example of its application. The radiation stress in, water waves is similar to that produced by electromagnetic or acoustic waves impinging on a surface; its normal component, $S_{x x}$ say, is defined as the mean (with respect to time) of the total flux of $x$-momentum across the plane $x=$ constant minus the mean flux in the absence of waves. For deep-water waves, Longuet-Higgins and Stewart (1964) showed that

$$
S_{x x}=\frac{1}{4} \rho g I^{2}=\frac{1}{2} E
$$

where $E$ is the total energy density.

The shear-radiation stress, $S_{y x}$ say, is the excess flux of $y$-momentum across $x=$ constant. For deep-water waves travelling in the $x$-direction, $s_{y x}=0$. Similarly, $s_{x y}=S_{y y}$ $=0$. Thus, the radiation-stress tensor for deep-water waves may be written:

$$
\mathbf{S}=\frac{1}{2} E\left[\begin{array}{ll}
1 & 0 \\
0 & 0
\end{array}\right] \text {. }
$$

We now generalize this expression to the obliquely incident ocean wave train considered in the previous section. This is done following the usual rules for tensor rotation, i.e. for a coordinate system inclined at an angle $\theta$ to the direction of propagation

$$
\mathbf{S}=\frac{1}{2} E\left[\begin{array}{cc}
\cos \theta & \sin \theta \\
-\sin \theta & \cos \theta
\end{array}\right]\left[\begin{array}{ll}
1 & 0 \\
0 & 0
\end{array}\right]\left[\begin{array}{cc}
\cos \theta & -\sin \theta \\
\sin \theta & \cos \theta
\end{array}\right],
$$

which reduces to

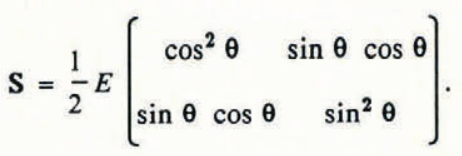

Thus, under conditions of total external reflection, i.e. when the angle of incidence is $\theta \geqslant \theta_{c}$, we have

$$
S_{x x}=\frac{1}{2} \rho g I^{2} \cos ^{2} \theta=E \cos ^{2} \theta,
$$

because for total reflection all the momentum is reversed, and

$$
S_{y x}=\frac{1}{4} \rho g I^{2} \sin \theta \cos \theta=\frac{1}{2} E \sin \theta \cos \theta .
$$

$S_{x x}$ and $S_{y x}$ represent the normal and shear force per unit distance along the ice edge due to totally reflected oblique ocean waves. In SI units, their dimensions are $\mathrm{N} \mathrm{m}^{-1}$.

\section{DATA}

The data used in this paper to compute the magnitude of the forces due to Equations (9) and (10) on the ice were collected from FS Polarstern during the Winter Weddell Sea Project of the austral winter of 1986. A full analysis of the data is under way and will be reported elsewhere. The interpretation of the data as showing total external reflection is novel and is inconclusive, principally because too few stations are available. However, as the data are used only as illustration that the mechanism might be important, we continue our story.

The experiment took place on either side of a consolidated band of ice about $4 \mathrm{~km}$ across. The seaward edge of the band ran along a bearing of about $060^{\circ}$. For the $24 \mathrm{~h}$ previous to the experiment, the wind originated in the $217-239^{\circ}$ sector. During the experiment the wind of about $11 \mathrm{~m} \mathrm{~s}^{-1}$ veered slightly from $210^{\circ}$ at the beginning to $245^{\circ}$ towards the end. Clear wave trains propagating in an approximately easterly direction could be discerned clearly on the ship's radar; fronts were timed and their average speed was found to be $20.4 \mathrm{~m} \mathrm{~s}^{-1}$ which corresponds to a period of $12.6 \mathrm{~s}$. Their easterly heading indicated that the wave energy was entering the band obliquely. This was also confirmed by observations from the ship's helicopter.

Ice concentration in the band was $100 \%$, composed of a slurry of pancake ice of average thickness approximately $0.25 \mathrm{~m}$. The mechanical behaviour of such a slurry is presumably highly complex and our assumption that it may be modelled as a thin elastic plate is questionable. Nonetheless, as we only require a change in the relative size of the wave number with period for the mechanism to be effective, the precise nature of the material characteristics of the ice band is unimportant.

Two directional wave-buoy recordings were made: the first seaward of the band (northerly station) and the second within the ice edge to the lee of the band (southerly station). In both, the I.O.S. pitch-roll buoy was used (Wadhams and others, 1986), and records were $4096 \mathrm{~s}$ long with a $2 \mathrm{~Hz}$ sample rate. After geo-orienting the data to north-south, east-west, the co- and quadrature spectra were derived and the energy density, the mean wave direction, and the angular spread were found as functions of wave frequency assuming a unimodal directional distribution. This is the conventional analysis for directional wave buoys first suggested by Longuet-Higgins and others (1963). All spectra were smoothed to 56 degrees of freedom. Figure 2 shows the energy spectrum and Figure 3 the wave direction and spread spectra, presented for completeness, both for the incident wave field (north) and for the wave field after it has passed through the band (south). Note the remarkable decrease in energy density across the band, particularly at short periods. It is this decrease which we hypothesize may, in part, be due to total external reflection at the ice edge To investigate the energy decrease further, we have plotted in Figure 4 the gain function between the incoming waves 


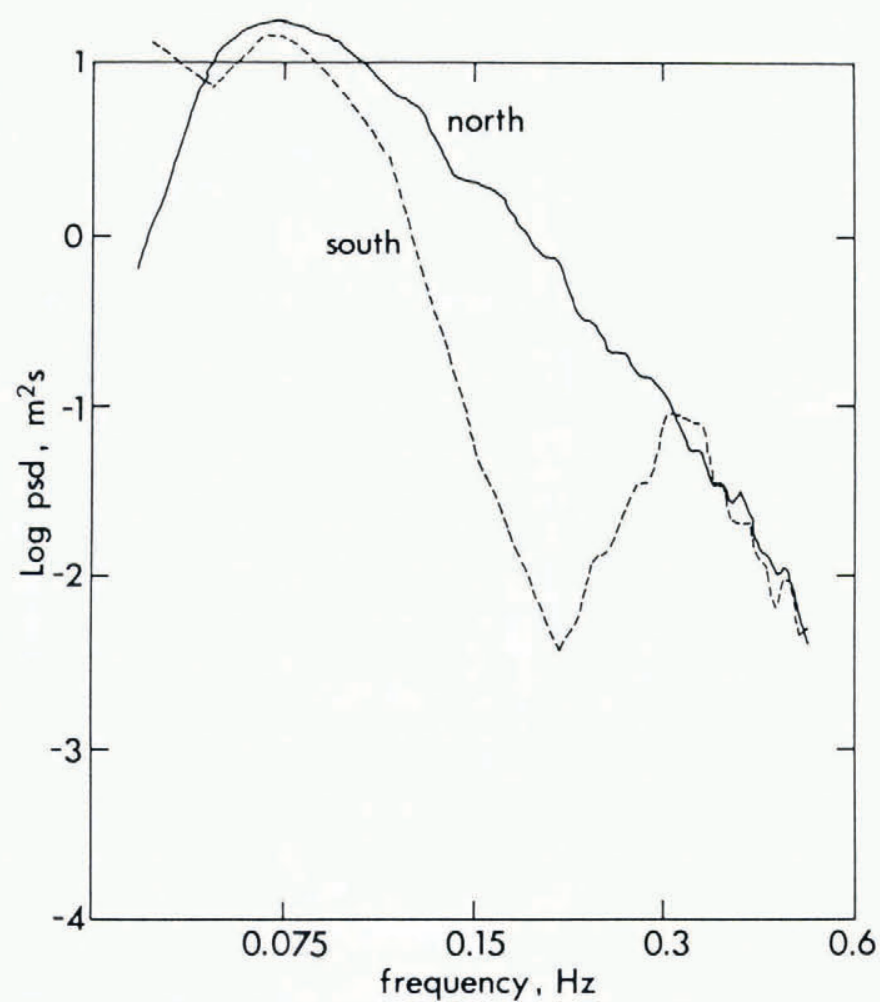

Fig. 2. The energy spectrum (power spectral density, psd) on either side of the ice band. The solid line (marked north) is for incoming waves, and the dashed line (marked south) is for waves after they have travelled through the band. The frequency axis has been plotted logarithmically so that the swell and wind sea peaks can easily be distinguished.

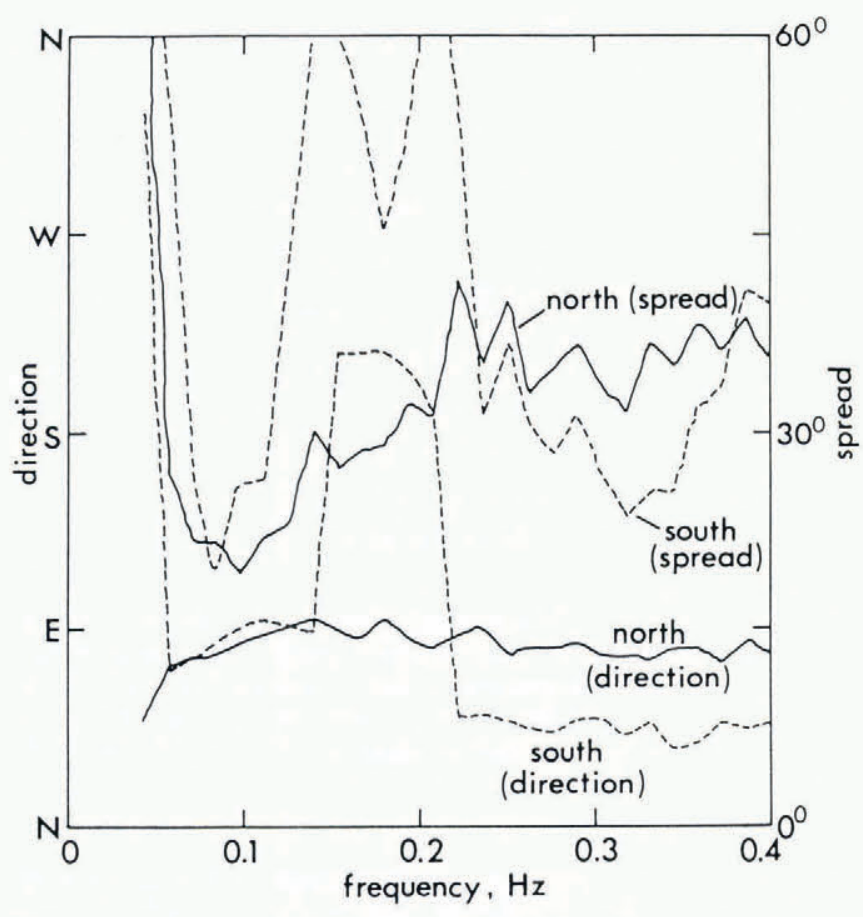

Fig. 3. The mean wave direction and spread spectra corresponding to Figure 2. The direction axis is the wave heading.

(northerly station) and the waves seen at the southerly station. The curve represents the fractional difference in amplitude as a function of wave frequency. Note the marked decrease in the gain function up to a frequency of $0.2 \mathrm{~Hz}$, followed by an increase for higher-frequency (lower-period) waves. The reason for this increase is unclear; certainly some short-period energy will be generated locally by winds. However, the regrowth begins to occur at a fairly long period for this mechanism alone to be responsible. Another possibility is multiple reflections from the next band encountered in the ice cover. We also note that the wave buoy was operating near to minimum resolution at the southerly station over the band width of frequencies surrounding the minimum of Figure 4.

In order to proceed, we must establish that the sea off the ice edge has a reflected component at some frequencies, i.e. that it is bimodal in its directionality. The method of analysis used above presumes the sea to be unimodal, and hence cannot reveal a reflected contribution. Any indication of wave reflection in the quadrature spectra between heave and pitch, and heave and roll, will be smeared out in the integration process used to derive mean wave direction by the conventional method:

$$
\text { Mean Wave Direction }=\tan ^{-1}\left[\frac{\int_{0}^{2 \pi} \sin \alpha G(\omega, \alpha) \mathrm{d} \alpha}{2 \pi}\right]
$$

where the function $G(\omega, \alpha)$ is the directional distribution which satifies

$$
\int_{0}^{2 \pi} G(\omega, \alpha) \mathrm{d} \alpha=1 \text {. }
$$

We therefore seek an alternative method for the evaluation of $G(\omega, \alpha)$ which does not presume a unimodal sea. One such method is the variational technique of Long and Hasselmann (1979) used successfully by Wadhams and others (1986). In the current work we shall use an alternative approach based on the maximum entropy algorithm suggested by Lygre and Krogstad (1986). This algorithm is easier to apply and is said to produce similar results to the variational technique (personal communication from J.A. Ewing, 1987). Sadly, Lygre and Krogstad's paper contains several typographical errors and care must be exercised in its application.

The maximum entropy method reproduces exactly all the Fourier coefficients that are input to the estimate. The elements of the usual cross-spectral matrix are used to derive the normalized angular harmonics $A_{1}, B_{1}, A_{2}$, and $B_{2}$ (see Wadhams and others, 1986, appendix A). Defining $c_{1,2}$ $=A_{1,2}+i B_{1,2}$, Lygre and Krogstad used the Yule-Walker maximum entropy equations to write down the following expression for $G(\omega, \alpha)$, valid at each radian frequency $\omega$ :

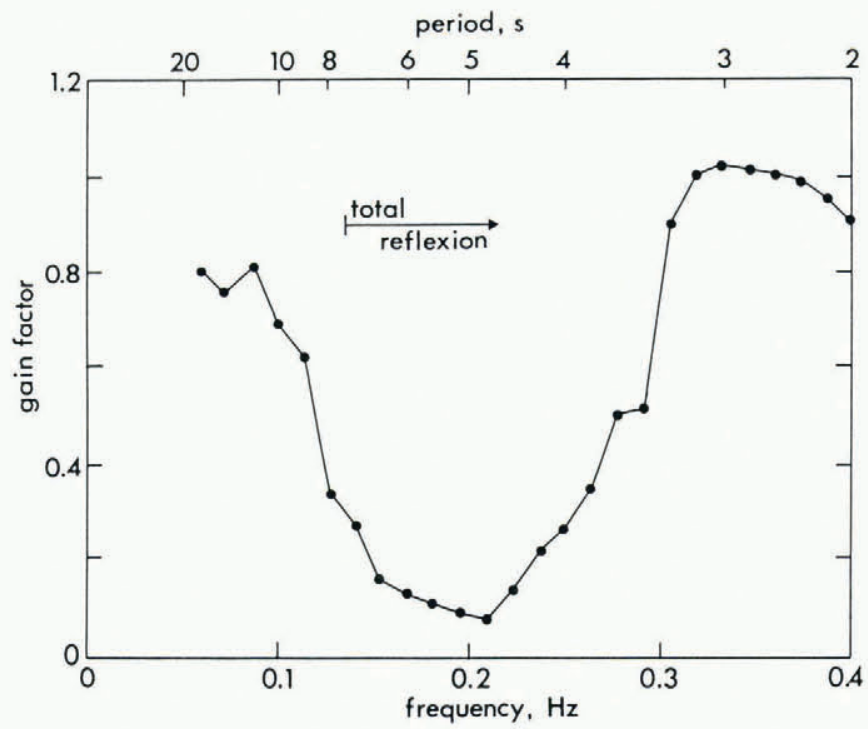

Fig. 4. The gain function between northerly and southerly wave stations. The frequency domain for which total reflection is possible is marked. 


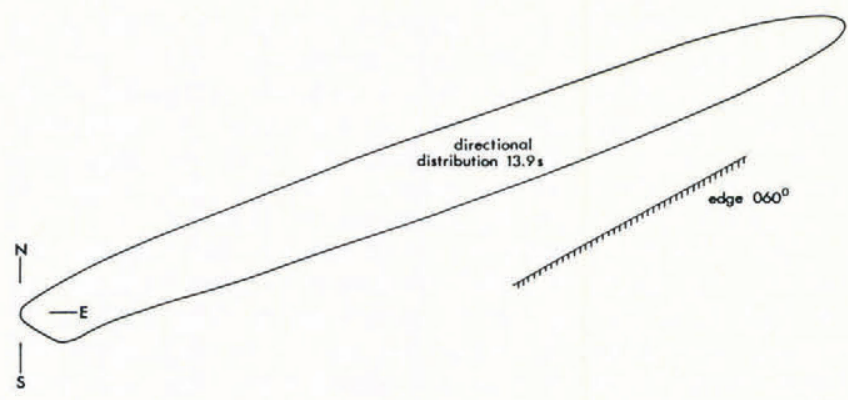

Fig. 5. Polar diagram of function $G(\alpha)$ at a period of $13.9 \mathrm{~s}$. The plot represents the angular distribution of energy at that period. No total external reflection occurs at the ice edge.

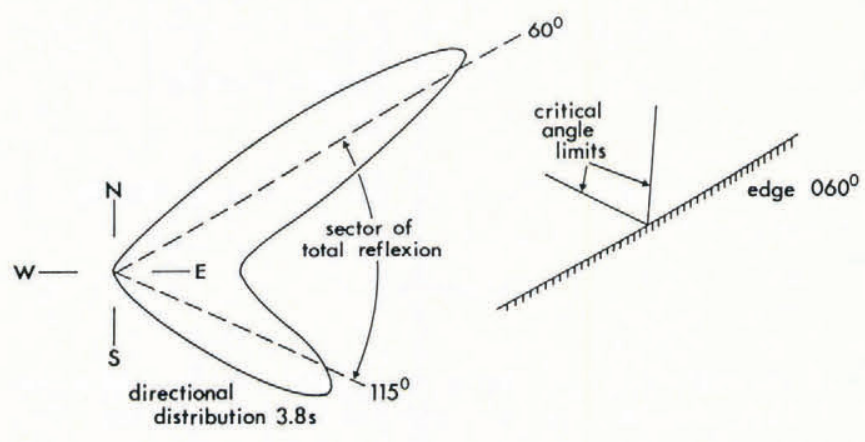

Fig. 6. Polar diagram of function $G(\alpha)$ at a period of $3.8 \mathrm{~s}$. The critical angle limits (determined from Figure 1) define a sector of total reflection between $060^{\circ}$ and $115^{\circ}$ at this period.

$$
2 \pi G(\alpha)=\frac{1-\phi_{1} c_{1}^{*}-\phi_{2} c_{2}^{*}}{\mid 1-\phi_{1} \mathrm{e}^{-i \alpha}-\phi_{2} \mathrm{e}^{-\left.2 i \alpha\right|^{2}}}
$$

where $\phi_{1}=\left(c_{1}-c_{2} c_{1}^{*}\right) /\left(1-\left|c_{1}\right|^{2}\right), \phi_{2}=\left(c_{2}-c_{1} \phi_{1}\right)$, and * indicates conjugate. Two examples of the directional distribution are given in Figures 5 and 6; the first figure shows that the waves are essentially unimodal at long period, and the second illustrates their bimodal nature at short periods. Processing was carried out on $4096 \mathrm{~s}$ records, smoothed to 56 degrees of freedom; resolution band width was therefore approximately $6.9 \times 10^{-3} \mathrm{~Hz}$. All periods in the spectral range plotted in Figure 2 were analysed. The change from bimodality to unimodality occurs between 7 and $8 \mathrm{~s}$.

Also in Figures 5 and 6 we have plotted the approximate line of the ice edge. This was obtained by taking ranges and bearings to the ice as seen by the radar aboard FS Polarstern and is hence subject to some error. Then the appropriate critical angle curve of Figure 1 has been used to delineate a sector wherein total external reflection is possible. The sector is bounded by a line parallel to the ice edge and a line determined by the relative magnitudes of $k$ and $k^{\prime}$. Since $k^{\prime}>k$ for long waves, no sector is defined in Figure 5 (13.9 s).

We are now in a position to compute the two forcedensity spectra suggested by Equations (9) and (10) for the normal and shear-radiation stresses, respectively. This is done as follows. For every period band present in the incoming wave spectrum (determined by our sampling and averaging constraints), the values of $S_{x x}$ and $S_{y x}$ are calculated for all incident angles in the sector defined in Figure 6 and its equivalent at different periods. Outside of the sector, of course, the radiation-stress contribution will vanish. The normal and shear force density per unit distance at each period along the ice edge is then the integral with respect to $\theta$ of these radiation-stress components around the sector. Their units will be $\mathrm{N} \mathrm{m}^{-1} \mathrm{~s}$. These values are plotted in Figure 7 as a spectrum for the

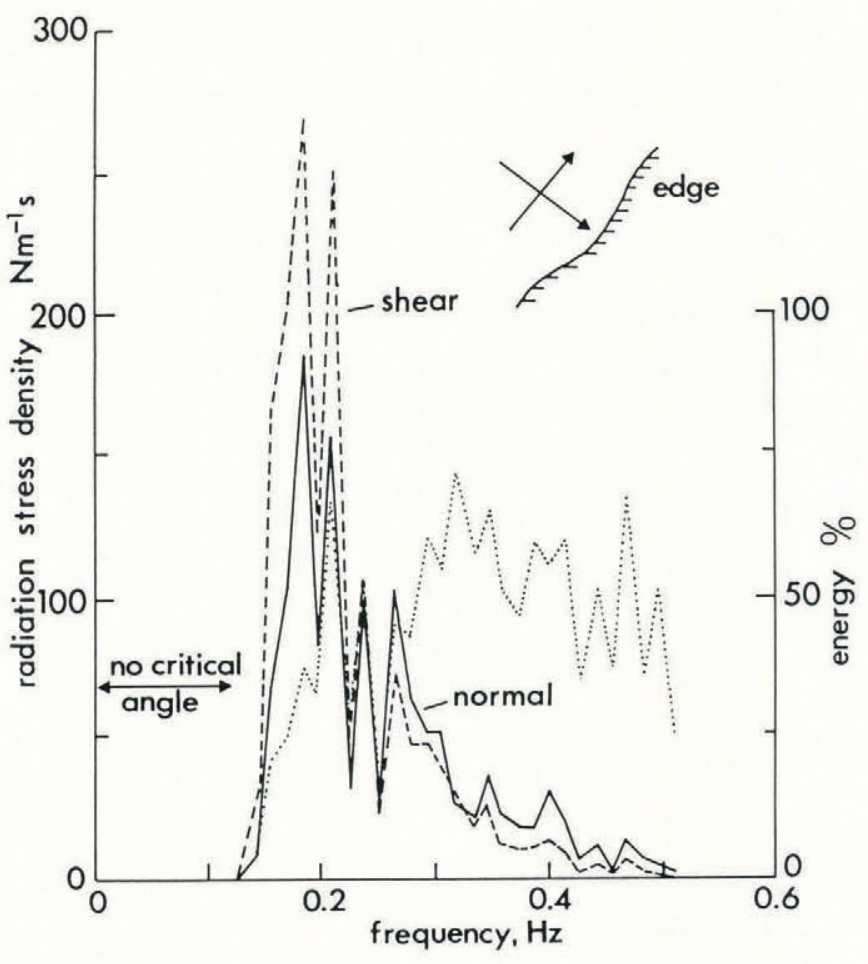

Fig. 7. Force-density spectra in the normal (solid line) and shear (dashed line) cases for an ice edge oriented at $060^{\circ}$. The percentage of the directional distribution of energy subject to possible reflection is also shown (dotted line). The graphs are only non-zero when total external reflection becomes possible.

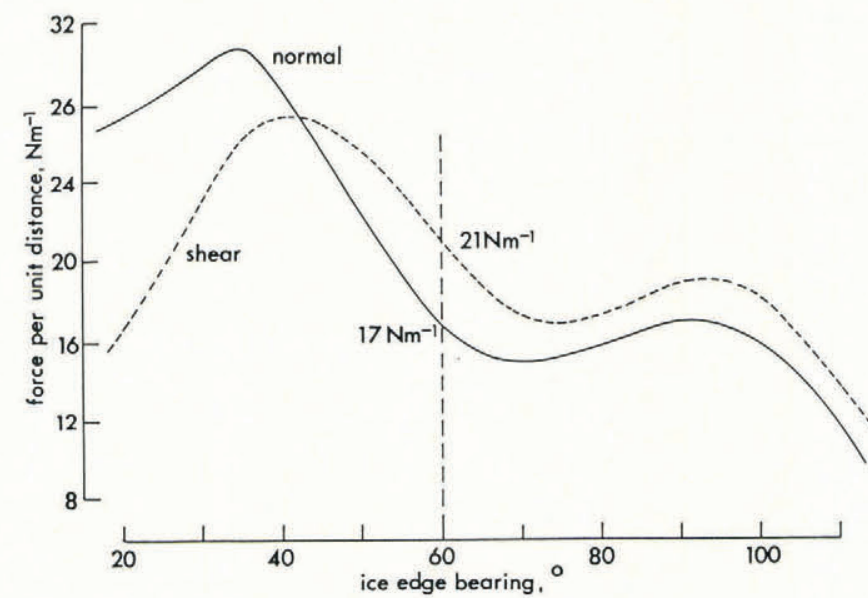

Fig. 8. Variation with ice-edge bearing of the total force per unit distance in the normal (solid line) and shear (dashed line) directions. The intersection of the radar-bearing ordinate $\left(060^{\circ}\right)$ and the curves gives a normal force of $17 \mathrm{~N}$ and a shear force of $21 \mathrm{~N}$ for each metre of ice edge.

normal and the shear cases. Also plotted is the percentage of the directional distribution which may be affected by total external reflection. Note the onset of reflection at about $0.12 \mathrm{~Hz}$.

The areas under the respective spectra of Figure 7 give the normal and shear force per unit distance exerted on the ice edge. In view of our uncertainty about the bearing of the ice edge, this has been calculated for various orientations of the ice edge. The results are presented in Figure 8. Note that, even with a change in orientation which is far beyond the likely error in the radar map, the two stresses change little. At the selected ice-edge bearing $\left(060^{\circ}\right)$ the normal force is about $17 \mathrm{~N} \mathrm{~m}^{-1}$ and the shear force is $21 \mathrm{~N} \mathrm{~m}^{-1}$. 


\section{SUMMARY AND CONCLUSIONS}

We have suggested that the dynamics of the ice edge may be influenced by an additional pair of forces which have hitherto not been considered or included in the momentum-balance equation. The forces derive from the excess flux of momentum in the incident wave's direction resolved into components into and along the ice edge for the case of total external reflection at the edge. We have tentatively assigned some recent Antarctic observations to this phenomenon.

Although the magnitude of the normal force on a $1 \mathrm{~m}$ strip of the ice edge $(17 \mathrm{~N})$ is less in this case than that imparted by the component of wind blowing across the entire width of the band $(22 \mathrm{~N}$, where we have assumed a drag coefficient of $\left.3 \times 10^{-3}\right)$, the figures are sufficiently close in view of our various approximations that the effect may be important. Moreover, for thicker sea ice and particularly for icebergs, total external reflection is more likely. Hence, in similar seas the two radiation stress-induced forces computed above would be larger and more significant, and could outweigh other terms in the momentum equation.

We conclude that the rather esoteric mechanism suggested in this paper may well prove an important factor in the control of the dynamics of the sea-ice edge and icebergs in the open ocean.

\section{ACKNOWLEDGEMENTS}

I congratulate $\mathrm{Dr} P$. Wadhams on his new appointment as Director of the Scott Polar Research Institute, University of Cambridge, England. The data presented in this paper were collected by the following team from the Scott Polar Research Institute: P. Wadhams (leader), the present author, S. Moore, R. Massom, M.P. Casarini. I thank the AlfredWegener-Institut für Polar- und Meeresforschung, the officers, crew, and fellow scientists of FS Polarstern, and the University of Otago for their support.

\section{REFERENCES}

Long, R.B. and K. Hasselmann. 1979. A variational technique for extracting directional spectra from multi-mode wave data. J. Phys. Oceanogr., 9, 373-381.

Longuet-Higgins, M.S. 1977. The mean forces exerted by waves on floating or submerged bodies with applications to sand bars and wave power machines. Proc. $R$. Soc. London, Ser. A, 352, 463-480.

Longuet-Higgins, M.S. and R.W. Stewart. 1964. Radiation stresses in water waves; a physical discussion, with applications. Deep-Sea Res., 11(4), 529-562.

Longuet-Higgins, M.S., D.E. Cartright, and N.D. Smith. 1963. Observations of the directional spectrum of sea waves using the motions of a floating buoy. In Ocean wave spectra. New York, Prentice-Hall, 111-136.

Lygre, A. and H.E. Krogstad. 1986. Maximum entropy estimation of the directional distribution of ocean wave spectra. J. Phys. Oceanogr., 16(12), 2052-2060.

Martin, S., P. Kauffman, and C. Parkinson. 1983. The movement and decay of ice edge bands in the winter Bering Sea. J. Geophys. Res., 88(C5), 2803-2812.

Muench, R.D., P.H. LeBlond, and L.E. Hachmeister. 1983. On some possible interactions between internal waves and sea ice in the marginal ice zone. J. Geophys. Res., 88(C5), 2819-2826.

Squire, V.A. 1984. On the critical angle for ocean waves entering shore fast ice. Cold Reg. Sci. Technol., 10(1), 59-68.

Squire, V.A. Unpublished. Dynamics of ocean waves in a continuous sea ice cover. (Ph.D. thesis, University of Cambridge, 1978.)

Wadhams, P. 1983. A mechanism for the formation of ice edge bands. J. Geophys. Res., 88(C5), 2813-2818.

Wadhams, P., V.A. Squire, J.A. Ewing, and R.W. Pascal. 1986. The effect of the marginal ice zone on the directional wave spectrum of the ocean. J. Phys. Oceangr., 16(2), 358-376. 